\title{
Evolution of radiographic joint damage in rituximab- treated versus TNF-treated rheumatoid arthritis cases with inadequate response to TNF antagonists
}

\author{
Axel Finckh, ${ }^{1,2}$ Burkhard Möller, ${ }^{3}$ Jean Dudler, ${ }^{4}$ Ulrich A Walker, ${ }^{5}$ Diego Kyburz, ${ }^{6}$ \\ Cem Gabay. ${ }^{1}$ on behalf of the physicians of the Swiss Clinical Quality Management for \\ Rheumatoid Arthritis
}

\begin{abstract}
- An additional material is published online only. To view these files, please visit the journal online (http://dx.doi.org/10.1136/ annrheumdis-2011-201016)

1Division of Rheumatology, University Hospital of Geneva, Geneva, Switzerland

2Division of Clinical

Epidemiology, University

Hospital of Geneva, Geneva,

Switzerland

${ }^{3}$ Department of Rheumatology, Clinical Immunology and Allergy, University Hospital of Bern, Bern, Switzerland

${ }^{4}$ Division of Rheumatology, Fribourg Hospital, Fribourg, Switzerland

${ }^{5}$ Department of Rheumatology, Basel University, Basel,

Switzerland

${ }^{6}$ Department of Rheumatology, University Hospital, Zurich, Switzerland
\end{abstract}

\section{Correspondence to \\ Dr Axel Finckh, Division of Rheumatology, University Hospital of Geneva, \\ 26 Av. Beau-Sejour, 1211 Geneva 14, Switzerland; axel.finckh@hcuge.ch}

Received 11 November 2011 Accepted 31 January 2012

\begin{abstract}
Background Observational studies have suggested that patients with rheumatoid arthritis (RA) who experience inadequate response to anti-tumour necrosis factor (anti-TNF) agents respond more favourably to rituximab (RTX) than to an alternative anti-TNF agent. However, the relative effectiveness of these agents on long-term outcomes, particularly in radiographic damage, remains unclear.
\end{abstract}

Objective To compare the effectiveness of RTX against anti-TNF agents in preventing joint damage in patients with RA who have experienced inadequate response to at least one prior anti-TNF agent.

Methods This is a prospective cohort study within the Swiss registry of patients with RA who discontinued at least one anti-TNF agent and subsequently received either RTX or an alternative anti-TNF agent. The primary outcome, progression of radiographic joint erosions (Ratingen erosion score) over time, and the secondary outcome, functional disability (Health Assessment Questionnaire Disability Index), were analysed using regression models for longitudinal data and adjusted for potential confounders.

Results Of the 371 patients included, 104 received RTX and 267 received an alternative anti-TNF agent. During the 2.6-year median follow-up period, the rates of Ratingen erosion score progression were similar between patients taking RTX and patients taking an alternative anti-TNF agent ( $p=0.67)$. The evolution of the Health Assessment Questionnaire score was statistically significantly better in the RTX group ( $p=0.016)$, but the magnitude of the effect was probably not clinically relevant.

Conclusion This observational study suggests that RTX is as effective as an alternative anti-TNF agent in preventing erosions in patients with RA who have previously experienced inadequate response to anti-TNF agents.

\section{INTRODUCTION}

Over the last decade, remarkable advances in the treatment of rheumatoid arthritis (RA) have been achieved, mostly owing to new anti-rheumatic treatments. The current anti-rheumatic armamentarium in RA includes several synthetic diseasemodifying anti-rheumatic drugs (DMARDs) and nine approved biological agents. However, more choices also lead to new challenges. One of these challenges is selecting the best treatment for an individual patient and pondering the potential benefits against the possible harms of a particular intervention in a given clinical setting. A recent conference aimed to identify major gaps in our current clinical knowledge of RA management and listed 'the comparison of active anti-rheumatic treatment options in patients for whom at least one tumour necrosis factor (TNF) inhibitor has failed' as one of the key areas for clinical investigation. ${ }^{1}$

Comparative effectiveness research in RA is still in its infancy; the positioning of newer biological agents, in particular, has not been fully established. ${ }^{2}$ The only published randomised controlled trial (RCT) to indirectly compare two biological agents has been the ATTEST trial ('Abatacept or infliximab versus placebo, a Trial for Tolerability, Efficacy and Safety in Treating RA'), ${ }^{3}$ which evaluated a T cell costimulation blocker against a TNF inhibitor (anti$\mathrm{TNF}$ ) in patients who have failed methotrexate treatment. Lacking head-to-head trials comparing biological agents, we have used observational studies to examine comparative effectiveness despite their susceptibility to selection biases and confounding factors. In particular, several cohort studies have analysed the effectiveness of switching to a second anti-TNF agent, compared to switching to a biological agent with a different mechanism of action, in patients who have experienced inadequate response to previous anti-TNF agents. ${ }^{4-10}$ A meta-analysis concluded that switching to rituximab (RTX) was slightly more effective than maintaining drug class by switching to a second anti-TNF agent in reaching American College of Rheumatology 70\% improvement criteria or a disease activity score remission response. ${ }^{11}$ Some studies have suggested that the relative benefit of RTX over an anti-TNF agent was restricted to patients switching due to the ineffectiveness of prior anti-TNF agents, but published results are essentially limited to only short-term outcomes such as RA disease activity. Long-term outcomes, such as structural joint damage or disability, may however be more relevant to chronic conditions such as RA and remain a concern. Prevention of structural damage has been suggested as the gold standard for drug studies in RA. ${ }^{12}$ AntiTNF agents have demonstrated outstanding efficacy in preventing radiographic joint damage even when 
Table 1 Baseline patient and treatment characteristics

\begin{tabular}{|c|c|c|c|}
\hline Disease characteristics & RTX (n=155) & $\begin{array}{l}\text { Alternative anti-TNF } \\
\text { agent }(n=163)\end{array}$ & $\mathbf{p}$ \\
\hline Age (years), median (IQR) & $58(47-66)$ & $56(44-64)$ & 0.15 \\
\hline Male sex (\%) & 25 & 19 & 0.18 \\
\hline $\operatorname{ACPA}(\%)^{*}$ & 81 & 74 & 0.30 \\
\hline RF (\%) & 92 & 82 & 0.01 \\
\hline Disease duration (years), mean (SEM) & $12(0.8)$ & $11(0.5)$ & 0.13 \\
\hline Disease activity (DAS28), mean (SEM) & $4.7(0.14)$ & $4.2(0.08)$ & 0.003 \\
\hline \multicolumn{4}{|l|}{ Radiographic erosion score } \\
\hline Ratingen erosion score (0-190), mean (SEM) & $34.9(3.2)$ & $32.5(2.3)$ & 0.64 \\
\hline ERO\%, mean (SEM) & $18.1(1.7)$ & $17.1(1.2)$ & 0.64 \\
\hline Health Assessment Questionnaire (0-3), mean (SEM) & $1.27(0.07)$ & $1.13(0.04)$ & 0.07 \\
\hline Concomitant use of DMARDs (\%) & 74 & 79 & 0.30 \\
\hline Methotrexate (\%) & 34 & 46 & 0.03 \\
\hline Leflunomide (\%) & 14 & 20 & 0.15 \\
\hline Other DMARDs $(\%) \dagger$ & 8 & 9 & 0.61 \\
\hline Glucocorticoids (\%) & 56 & 48 & 0.16 \\
\hline \multicolumn{4}{|l|}{ Previous anti-TNF agents (n) } \\
\hline Median (IOR) & $1(1-2)$ & $1(1-1)$ & $<0.001$ \\
\hline Mean (SD) & $1.43(0.6)$ & $1.01(0.12)$ & $<0.001$ \\
\hline $\begin{array}{l}\text { Time since the discontinuation of previous anti-TNF agent } \\
\text { (months), median (IQR) }\end{array}$ & $1(0.1-4.0)$ & $1.8(0.5-13.6)$ & 0.004 \\
\hline \multicolumn{4}{|c|}{$\begin{array}{l}\text { Percentages indicate the use of each cotreatment at baseline. Patients could receive more than one DMARD cotreatment, explaining } \\
\text { why the sum of individual DMARDs may exceed } 100 \% \text {. } \\
{ }^{*} \text { Available for only half of all patients. } \\
\text { tOther DMARDs included synthetic DMARDs such as hydroxychloroquine, sulfasalazine and azathioprine. } \\
\text { ACPA, anti-citrullinated protein antibody; DAS28, Disease Activity Score based on } 28 \text { joints; DMARDs, disease-modifying anti- } \\
\text { rheumatic drugs (including oral glucocorticoids); ERO \%, Ratingen erosion score expressed in per cent maximum erosion score; RF, } \\
\text { rheumatoid factor; RTX, rituximab; TNF, tumour necrosis factor; IQR, interquartile ranges; SEM, standard error of the mean. }\end{array}$} \\
\hline
\end{tabular}

the clinical response was not satisfactory, ${ }^{13}$ while inhibition of structural joint damage by RTX was initially perceived as less impressive, ${ }^{14}$ probably owing to different patient populations.

The aim of this analysis was to examine the effectiveness of switching to an alternative anti-TNF agent versus initiating RTX on long-term outcomes such as radiographic damage progression and functional disability. Both biological agents have established efficacy in preventing radiographic damage in placebo-controlled RCTs ${ }^{15} 16$ but have never been compared directly for their efficacy in this key outcome.

\section{METHODS}

Study design

We performed a nested cohort study to examine the impact of switching to an alternative anti-TNF agent versus RTX in patients with active RA and the impact of inadequate response to at least one previous anti-TNF agent. The study's predefined primary outcome was evolution of joint erosions. Ethical approval for the enrollment of patients into the Swiss Clinical Quality Management (SCOM) programme and related studies was obtained from the Swiss Academy of Medical Sciences review board.

\section{Study population}

SCOM-RA is a Swiss cohort of patients with RA that has been described in detail elsewhere. ${ }^{17}$ Patients are assessed at regular intervals for disease activity, radiographic erosions, past and current anti-rheumatic treatments, reasons for changes in treatment, adverse events and RA symptoms. ${ }^{17}$ The Swiss regulatory authorities perform continuous monitoring of all patients with arthritis who are taking biological agents within the SCOM programme; therefore, the cohort can be considered a representative population-based sample of Swiss patients with RA having biotreatment. SCOM patients come from a range of clinical settings, with more than $50 \%$ enrolled by private practices, $30 \%$ from non-academic centres and $20 \%$ from academic centres. The analysis included data collected from March 1996 through November 2010. The estimated proportion of Swiss patients with RA having biotreatment was around $13 \%$ in 2008, in line with other western European countries. ${ }^{18}$

RTX has been approved for the treatment of moderate to severe cases of RA only after the failure of anti-TNF agents. We therefore restricted the eligibility criteria for this analysis to patients who discontinued at least one anti-TNF agent (infliximab, etanercept, adalimumab, golimumab or certolizumab) and subsequently initiated either an alternative anti-TNF agent or a first course of RTX. Thus, patients receiving anti-TNF or RTX treatment as their first biotreatment were excluded from the analysis. Other inclusion criteria were a diagnosis of RA by a board-certified rheumatologist and the availability of at least two consecutive sets of radiographs. Because radiographs should reflect structural joint damage occurring during the treatment of interest, we excluded radiographs that preceded the initiation of treatment by more than 6 months. The only exclusion criterion was RTX treatment for lymphoma.

The duration of drug exposure is not always clear-cut for biotreatments, particularly for RTX, and patients can be lost to follow-up after varying durations, affecting the period of drug exposure. ${ }^{19}$ Performing an 'on-drug-only' analysis or a 'completers-only' analysis (ie, including only the patients with at least two consecutive sets of radiographs taken during exposure to the drug of interest) carries the risk of overestimating the true treatment effect, as only patients with satisfactory responses to treatment will remain on treatment long enough to have two radiographic assessments. To avoid this bias, we operationally defined drug exposure at the initiation of the new treatment, whether or not patients continued to receive their initial treatment. This is a conservative approach comparable to an intent-to-treat analysis in randomised trials. In sensitivity analysis, we considered an alternative definition for drug exposure ('on drug only', with observations censored after treatment interruption) according to recently published recommendations. ${ }^{19}$ 


\section{Evolution of radiographic joint damage}

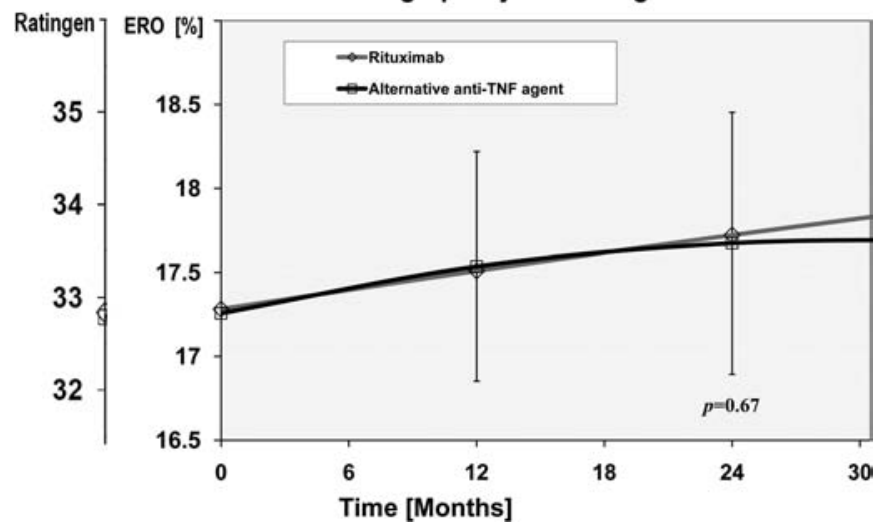

Figure 1 Evolution of radiographic damage after the initiation of an alternative tumour necrosis factor (TNF) inhibitor versus rituximab (RTX; open diamond with a red line across). The change in radiographic damage score (Ratingen erosion score and ERO\%) from baseline over the average follow-up period is represented for patients treated with RTX or with an alternative TNF inhibitor (alternative anti-TNF agent: open square with a black line across). The progression trajectories depicted are adjusted for baseline disease characteristics, in particular baseline ERO, disease activity and treatment characteristics. ERO\% expresses the Ratingen erosion score in per cent maximum damage score. Ratingen expresses the erosion score in the original scale (0-190). Vertical lines represent the $95 \% \mathrm{Cl}$ of the mean for the anti-TNF group. Alternative anti-TNF agent represents a second, third or fourth TNF inhibitor.

\section{Outcomes}

The primary end point of this analysis was radiographic erosion progression as measured by the change in radiographic scores from baseline. A validated scoring method (Ratingen erosion score) ${ }^{20}$ was used to grade serial radiographs according to the number and the size of bone erosions. The Ratingen scoring method has good reliability, with an intrarater intraclass correlation coefficient (ICC) of $0.8-0.9$ and an inter-rater ICC of 0.7-0.9, and is less susceptible to ceiling effects in advanced disease because of a true ordinal rating system. ${ }^{20} 21$ The minimal detectable radiographic change for this method has been determined to be around $3.3 \%$ of the maximum score. ${ }^{20}$ Radiographic damage was assessed prospectively by two independent assessors who were blinded to clinical information. Their reliability was good, with an intrareader ICC of 0.83 and an inter-rater ICC of between 0.83 and $0.96 .^{22}$ The average of the two assessors' change scores was used for the analysis. Ratingen erosion score can be expressed both in the original unit (Ratingen, range 0-190) and in per cent maximum damage score (ERO\%).

A secondary study end point was the evolution of functional disability, measured as a change in the Health Assessment Questionnaire Disability Index (HAO-DI) from baseline. ${ }^{23}$ The Health Assessment Questionnaire (HAQ) score ranges from 0 to 3 , with 3 representing the maximum possible disability. Furthermore, important baseline predictors of RA disease progression, such as demographic characteristics, various disease characteristics, concomitant treatment with DMARD and selfassessed questionnaires, were extracted from the SCOM-RA database and used to assess the comparability of the patient groups and to adjust the analysis.

\section{Analysis}

Based on a previous analysis, we calculated that 320 patients (with a 2:1 ratio of anti-TNF-agent-treated patients to RTXtreated patients) would be required to demonstrate a similar

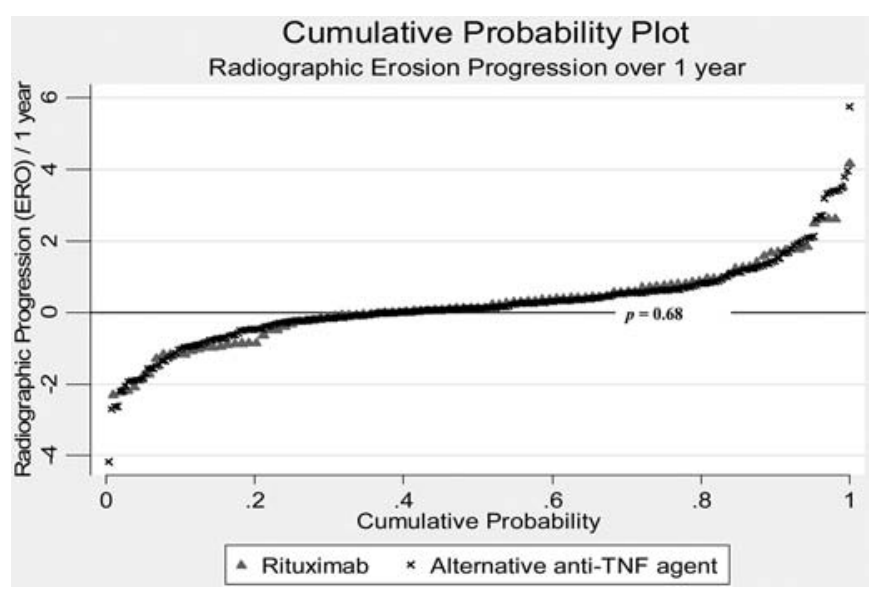

Figure 2 Probability plot of radiographic damage progression with alternative tumour necrosis factor inhibitors versus rituximab. Probability plot of the individual rates of radiographic damage progression over 1 year with the two drugs. The $\mathrm{X}$ axis displays cumulative probability, and the $Y$ axis displays the rate of radiographic damage progression over 1 year. Radiographic progression is assessed using the Ratingen erosion score expressed in per cent maximum damage score (ERO\%). The horizontal line represents the absence of progression.

difference in radiographic progression, ${ }^{24}$ a type I error probability of 0.05 and a statistical power of $80 \%$. Baseline disease and treatment characteristics were compared using conventional descriptive statistics. The significance of differences in baseline disease characteristics was assessed with Student t test for normally distributed mean values of continuous variables and with Kruskal-Wallis test for non-normally distributed variables. For dichotomous variables, Pearson's chi-square test was used to evaluate the statistical significance of differences in proportions. Drug retention was examined using survival analysis, and differences in drug survival were explored with a Cox proportional hazard model. In order to minimise potential biases induced by sporadically missing covariates $(\leq 5 \%)$, we used the population average as substitute. Statistical tests were two-sided and were evaluated at the 0.05 significance level. The analysis was performed with STATA V. 11 (Stata Statistical Software, College Station, Texas, USA).

In observational studies, selection bias is always of concern because assignment to RTX versus alternative TNFs is not perfomed randomly. Because differences in disease characteristics may substantially influence subsequent radiographic progression, we used multivariate regression models to adjust for potential confounding factors. The final adjusted model was adjusted for age, sex, rheumatoid factor (RF) positivity, disease duration, functional disability (HAQ), disease activity (Disease Activity Score based on 28 joints (DAS28)), time since discontinuation of previous anti-TNF agent, radiographic damage at baseline, use of low-dose glucocorticoid, concomitant use of synthetic DMARD, number of prior anti-TNF agent failures and primary failure of the previous anti-TNF agent (defined as discontinuation within the first 6 months). The evolution of radiographic erosions or functional disability outcomes was analysed using generalised mixed models for longitudinal data. We first selected the best-fitting model without controlling for potential confounders. We then adjusted the analysis for differences in baseline disease characteristics using a multivariate model (adjusted model). We explored potential effect modification by concomitant DMARD treatment, concomitant glucocorticoid treatment, RF positivity and primary failure of the previous anti-TNF agent 
Evolution of Functional Disability (HAQ)

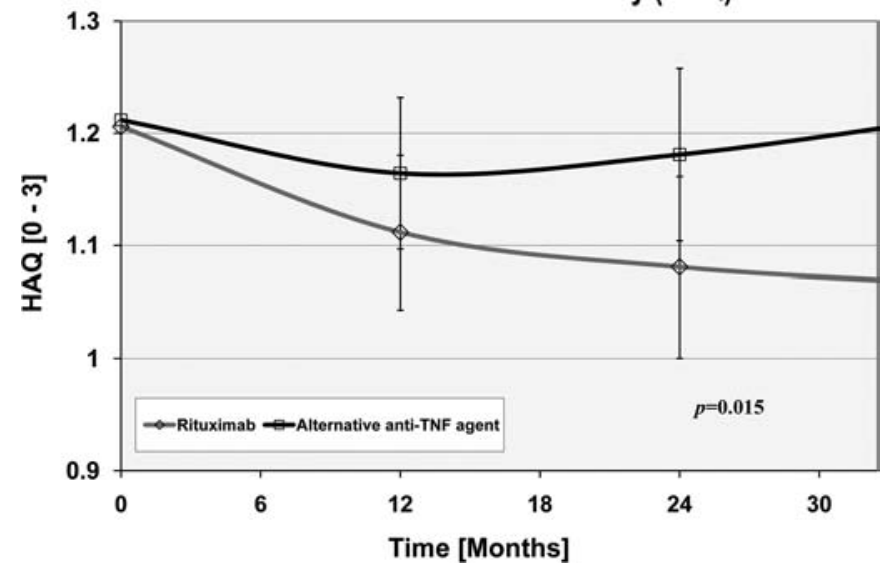

Figure 3 Change in functional disability after the initiation of an alternative tumour necrosis factor (TNF) inhibitor versus rituximab (RTX). The change in functional disability (Health Assessment Questionnaire Disability Index (HAQ-DI)) from baseline over the average follow-up period is represented for patients treated with RTX (open diamond with a red line across) or with an alternative TNF inhibitor (alternative anti-TNF agent: open square with a black line across). The progression trajectories depicted are adjusted for baseline disease characteristics, baseline HAO-DI scores and treatment characteristics. Vertical lines represent the $95 \% \mathrm{Cl}$ of the mean for the anti-TNF group. Alternative anti-TNF agent represents a second, third or fourth TNF inhibitor.

using interaction terms. We produced a cumulative probability plot with the individual rates of radiographic progression over a 1 -year period based on the above model estimates.

\section{RESULTS}

A total of 650 patients with RA in the SCOM-RA database were taking an alternative anti-TNF agent or RTX after an initial inadequate response to anti-TNF agents and had at least one radiographic assessment while having this treatment. Of these, 371 patients $(57 \%)$ had subsequent radiographic assessments, leaving available for evaluation 104 patients taking RTX and 267 patients taking an alternative anti-TNF agent. The patients excluded from the analysis initiated their biotreatment significantly later $(1.9$ years, $\mathrm{p}<0.001)$ but had otherwise mostly similar disease characteristics (supplemental file), suggesting that missing follow-up time was the main reason for the absence of subsequent radiographs in these patients. The 371 patients matching the inclusion criteria had means of 2.8 radiographic assessments and 4.7 HAQ assessments during a median followup period of 2.6 years (IOR 1.6-3.6). The median period of drug retention ('drug survival') was 3.43 years, which was not significantly different between the two groups ( $p=0.16$ ).

Patients taking RTX received a median number of two cycles of RTX (a cycle consisting of two infusions of $1000 \mathrm{mg}$ each, with concomitant use of glucocorticoids) in accordance with the manufacturer's recommendations. Fifty-one per cent of patients taking an alternative anti-TNF agent received adalimumab (40 mg subcutaneously every 2 weeks), $25 \%$ received etanercept (50 $\mathrm{mg}$ per week), $16 \%$ received infliximab ( $3 \mathrm{mg} / \mathrm{kg}$ intravenously) and $8 \%$ received either golimumab or certulizumab. Some differences between the RTX group and the alternative anti-TNF group were noted (table 1). Combination with methotrexate was more frequent in patients treated with an anti-TNF agent than in patients treated with RTX, but overall DMARD use was not significantly different between the two groups. Patients treated with RTX were more often positive for RF or anti-citrullinated protein antibody, had higher baseline DAS28 levels (4.7 vs 4.2), had a shorter interval between discontinuing the prior antiTNF agent and starting the new biotreatment (1 vs 1.8 months) and failed more prior anti-TNF agents (1.4 vs 1). RTX was the second biological agent in $52 \%$ of patients, the third biological agent in $39 \%$ of patients and the fourth biological agent in $9 \%$ of patients; anti-TNF agent was the second biological agent in $80 \%$ of patients, the third biological agent in $18 \%$ of patients and the fourth or fifth biological agent in $2 \%$ of patients. Other important prognostic markers for radiographic progression, in particular baseline radiographic erosion scores and functional disability (HAO) scores, were similar.

While the evolution of disease activity (DAS28) was significantly better in the RTX-treated group (at 1 year, incremental DAS28 improvement $-0.34(95 \% \mathrm{CI}-0.14$ to -0.53$)$ ) as previously demonstrated, ${ }^{4}$ the longitudinal evolution of radiographic erosions was similar between the RTX group and the alternative anti-TNF group ( $\mathrm{p}=0.67$; figure 1). Overall, ERO \% progressed, on average, by $0.23 \%$ (95\% CI -0.22 to +0.69 ; Ratingen $+0.44(95 \%$ CI -0.42 to +1.31$)$ ) during the first year, representing approximately one new erosion in 2 years of follow-up or a low level of damage progression. ${ }^{20}$ ERO \% progression with an alternative anti-TNF agent was $0.25 \%(95 \%$ CI -0.06 to +0.57 ; Ratingen $+0.48(95 \%$ CI -0.11 to +1.08$))$ during the first year after the switch, compared to $0.23 \%$ ( $95 \%$ CI -0.41 to +0.88 ; Ratingen $+0.44(95 \%$ CI -0.76 to +1.67$))$ with RTX. This means that $33 \%$ (34/104) of all patients taking RTX have presented at least one new erosion over a period of 1 year compared to $30 \%(81 / 266)$ of patients taking an alternative anti-TNF agent $(p=0.68$; figure 2). Significant predictors of subsequent radiographic progression were baseline ERO\% level and high disease activity. We found no effect modification by concomitant use of DMARD ( $p=0.18)$, use of low-dose oral glucocorticoid use $(p=0.26)$, RF seropositivity $(\mathrm{p}=0.79)$ or a personal history of primary anti-TNF agent failure (0.20). A sensitivity analysis with an alternative definition of drug exposure ('on drug only') did not qualitatively change the conclusions (supplemental file).

The longitudinal evolution of functional disability was statistically significantly better in the RTX group compared to the alternative anti-TNF group ( $\mathrm{p}=0.015$; figure 3 ); however, the difference was far from reaching a minimally clinically important difference, estimated to be at least $0.22 \mathrm{HAQ}-\mathrm{DI}$ units. ${ }^{25}$ The HAQ-DI score improved by $0.05 \mathrm{HAQ}$ units $(95 \% \mathrm{CI}-0.017$ to +0.11 ) more on RTX 1 year after the switch and by $0.14 \mathrm{HAO}$ units (95\% CI 0.04 to 0.25$) 3$ years after the switch. Significant predictors for functional deterioration were high baseline levels of functional disability $(p<0.001)$, older age $(p=0.03)$, high disease activity $(p=0.03)$ and a trend for a more favourable evolution with concomitant use of steroid ( $p=0.07$ ).

\section{DISCUSSION}

In the absence of any trial directly comparing the efficacy of RTX and an alternative anti-TNF agent, ${ }^{26}$ we analysed the effectiveness of switching to an alternative anti-TNF agent versus RTX on long-term outcomes in a longitudinal cohort study. After adjusting for potential confounders, we found no significant differences in the rates of erosion progression between patients taking an alternative anti-TNF agent and patients taking RTX. The longitudinal progression of functional disability (HAQ) suggested an advantage for patients treated with RTX compared to patients treated with an alternative anti-TNF agent.

Previous observational studies have generally suggested that biological agents with a different mechanism of action, such as 
RTX, are more effective on disease activity than an alternative anti-TNF agent in patients with RA who experienced inadequate response to a previous anti-TNF agent. ${ }^{4-11}$ However, there has been a concern that RTX might not be as effective in preventing radiographic damage as anti-TNF agents. While we could confirm the relative benefit of RTX over alternative anti-TNF agents on disease activity, we found a similar rate of radiographic erosion progression between these two treatments. Given the established disconnect between inflammation control and radiographic damage progression with biological agents, ${ }^{13}$ this is not surprising. However, because of the limited sample size and follow-up, we cannot exclude the possibility that minor differences could appear over time, yet large differences in radiographic erosion progression between these agents appear very unlikely. We found no evidence for an effect modification by RF seropositivity, in contrast with what has been suggested for RTX. ${ }^{27}$ The improved effectiveness of RTX in seropositive patients has been established only for disease activity but not for radiographic progression or compared to other biological agents. Comparing our results to those of RCTs is difficult, as they involve very different patient populations, treatment designs and scoring methods. Nevertheless, our results confirm the effectiveness of these biological agents in halting radiographic disease progression, independently of their impact on disease activity.

The difference in the evolution of functional disability was unexpected, as functional disability in RA is thought to be related to underlying structural joint damage. However, disease activity may greatly influence the assessment of functional capacity particularly in early disease, ${ }^{28}$ which would explain the beneficial trend in the RTX group, as this agent has a more favourable impact on RA disease activity in this setting. ${ }^{4-11}$

Observational studies have inherent limitations when comparing the effectiveness of different treatment options. Selection bias may arise because assignment to these agents is not performed randomly. In fact, patients taking RTX were more often RF 'seropositive', had higher DAS28 levels at baseline and had failed more biotreatments before switching. While we could adjust for these differences using multivariate regression models, we cannot exclude some degree of residual confounding or confounding by unmeasured factors such as a carryover effect of the previous anti-TNF agent. However, major selection bias is unlikely, as the choice of an alternative biotreatment is currently still essentially a matter of the physician's personal preference (there are no 'treatment guidelines' for Swiss rheumatologists) and can thus be considered largely at random. The primary end point of this analysis was radiographic erosion progression as measured by the Ratingen scoring method, ${ }^{20}$ which implies that we could not assess the relative benefit of these treatments on joint space narrowing or cartilage degradation. Having incomplete data is another limitation of observational studies (ie, anti-citrullinated protein antibody status was available for only $50 \%$ of patients). Of all patients receiving an alternative biological agent after a prior inadequate response to an anti-TNF agent in the SCOM-RA cohort, $43 \%$ did not have at least two sets of consecutive radiographs. Excluded patients initiated their biotreatment significantly later, suggesting that missing radiographs were mainly due to insufficient follow-up time and were not related to treatment outcome. To further limit the potential for bias by selective loss to follow-up, we chose to define drug exposure at baseline, independently of whether patients remained on treatment (ie, intentto-treat analysis). Overall drug retention was not different in the two groups of patients. This study was not powered to examine safety parameters, which were not examined. Furthermore, it is difficult to determine whether the biological agents have been used optimally. Some patients might have benefitted from an earlier retreatment with RTX or from higher doses of anti-TNF agents. In particular, RTX retreatment is frequently delayed as most physicians still wait for a disease flare prior to administering another cycle of RTX. However, these results represent 'realworld' clinical practice with these agents.

After the failure of an anti-TNF agent, the rationale for switching to biological agents with a different mode of action is to surmount problems related to class. In patients who have persistent active disease despite anti-TNF treatment, previous studies suggested that a biological agent with a different mechanism of action may be more effective in controlling disease activity; however, this does not appear to result in a significant difference in joint erosions. Overall, our data suggest that RTX is at least equivalent to alternative anti-TNF agents on long-term outcomes of RA. In conclusion, these results indicate that a biological agent with a different mechanism of action, such as RTX, can be a very effective therapeutic alternative for patients who have responded inadequately to previous anti-TNF treatments.

Contributors AF and CG contributed to the research idea. AF performed statistical analysis. AF, BM, JD, UAW, DK and CG provided critical inputs to the intellectual content of the paper. AF, BM, JD, UAW, DK and CG wrote and edited the manuscript. AF, BM, JD, UAW, DK and CG performed data collection.

Acknowledgements The authors are grateful to the SCOM staff for data management and support and to the participating physicians and patients who made this study possible. The authors thank Guillaume Wuilleret for the second scoring of radiographs. The SCOM received grants from the Swiss health authorities (Bundesamt für Gesundhei), the Swiss Academy for Medical Sciences, the J L Warnery Foundation, the Swiss Society of Polyarthritic Patients and various pharmaceutical companies (Abbott, Pfizer, MSD, Roche, Bristol-Myers Squibb, Mepha, Novartis, Sanofi-Aventis and UCB). The authors specifically thank those rheumatologists who enrolled these patients (>10): Aellen, Nyon; Badaracco, Lugano; Bosia, Locarno; Bloesch, Lausanne; Bötschi, Romanshorn; Carey-Berner, Lausanne; Chamot A-M, Morges; Eigenmann, Zurich; Exer P, Basel; Forster A, Diessenhofen; Elmiger, Bern; Gäumann, Murten; Gerny, Thun; Gratzk, Basel; Häfelin, Schlieren; Gut, Reinach; Jaccard, Genolier; Kaiser, Thalwil; Kloti R, Brugg; Kowalski, Solothurn; Leuba-Manueddu, Neuchâtel; Lehmann T, Bern; Maager, Aarau; Maclachlan, Heiden; Mathieu, Solothurn; Martin, Liestal; Masina, Lugano; Meder, Zofingen; Meierhofer, Zurich; Morell, Schaffhausen; Muff Affoltern a. Albis; Müller-Werth, Sarnen; Marbet Grierson, Olten; Messikommer, Visp; Pancaldi, Muralto; Pfister, Bulach; Raccaud, Lausanne; Schönbächler, Zurich; Schneeberger, Martigny; Schwarz, Carouge; Schurch, Lausanne; Suter, Bern; von Mühlenen I, Basel; Wicht, Solothurn; Rheumatologie im Silberturm, St Gallen; Rheumazentrum Kreuzlingen; Rheumapraxis Männerdorf; Rösler, Bern; Sauvain, Fribourg; Tauxe, Vevey; Tinner, Weinfelden; Volken, Sierre; Widrig-Bernhardt, St Gallen; Wuest, Wädenwil; Zichmann, Zurich; Zuffrey, Estavayer Le Lac. The authors also especially thank the participating rheumatology clinics that registered these cases (>20): the rheumatology divisions of the University Hospital of Zurich; the University Hospitals of Geneva; the University Hospital of Vaud; the University Hospital of Bern; Bethesda Spital, Basel; Felix Platter Spital, Basel; Cantonal Hospital Aarau; Cantonspital St Gallen; Cantonal Hospital Luzern; Cantonal Hospital Wintherthur; Burgerspital Solothurn; Schulthess Klinik, Zurich; Hirslanden Klinik St Ana, Luzern; Stadtspital Triemli, Zurich; Clinic Impuls, Wetzikon; Cantonal Hospital Schaffhausen; Immunologie-Zentrum, Zurich; RehaClinic Zurzach; AaReha Schinznach, Cantonal Hospital Zug; Hirslanden Clinic Birshof, Münchenstein; Cantonal Hospital Fribourg.

Collaborators $0 n$ behalf of the physicians of SCQM-RA.

Funding Swiss National Science Foundation (and an unrestricted research grant from Roche)

Competing interests AF discloses board memberships or payment for lectures for Abbott, BMS, Pfizer, Roche, and Schering- Plough. BM discloses board memberships or grants from Abbott, BMS, Pfizer and Roche. JD discloses consultancies or payment for lectures for Abbott, BMS, MSD, Pfizer, Roche and UCB. UW discloses board memberships or payment for lectures for MSD, Pfizer, Roche and UCB. CG discloses board memberships, consultancies or payment for lectures for Abbott, BMS, MSD, Pfizer, and Roche. DK discloses board memberships, grants or payment for lectures for Abbott, Amgen, BMS, MSD, Pfizer, UCB, and Vifor Pharma.

Ethics approval Swiss Academy of Medical Sciences review board.

Provenance and peer review Not commissioned; externally peer reviewed. Data sharing statement The original data for this research are kept in the SCOM registry. 


\section{REFERENCES}

1. Rheumatoid Arthritis Clinical Trial Investigators Ad Hoc Task Force. American College of Rheumatology clinical trial priorities and design conference, July 22-23, 2010. Arthritis Rheum 2011;63:2151-6.

2. Finckh A. Comparative effectiveness of rheumatoid arthritis therapies. Curr Rheumatol Rep 2010;12:348-54.

3. Schiff M, Keiserman M, Codding C, et al. Efficacy and safety of abatacept or infliximab vs placebo in ATTEST: a phase III, multi-centre, randomised, double-blind, placebo-controlled study in patients with rheumatoid arthritis and an inadequate response to methotrexate. Ann Rheum Dis 2008;67:1096-103.

4. Finckh A, Ciurea A, Brulhart $L$, et al. B cell depletion may be more effective than switching to an alternative anti-tumor necrosis factor agent in rheumatoid arthritis patients with inadequate response to anti-tumor necrosis factor agents. Arthritis Rheum 2007;56:1417-23.

5. Finckh A, Ciurea A, Brulhart $L$, et al. Which subgroup of patients with rheumatoid arthritis benefits from switching to rituximab versus alternative anti-tumour necrosis factor (TNF) agents after previous failure of an anti-TNF agent? Ann Rheum Dis 2010;69:387-93.

6. Blom M, Kievit W, Donders AR, et al. Effectiveness of a third tumor necrosis factor$\alpha$-blocking agent compared with rituximab after failure of 2 TNF-blocking agents in rheumatoid arthritis. J Rheumatol 2011;38:2355-61.

7. Emery $\mathbf{P}$, Sarzi-Puttini P, Moots R, et al. Relative effectiveness of rituximab and an alternative TNF inhibitor in patients with rheumatoid arthritis who had experienced an insufficient response or intolerance to a single previous TNF inhibitor: interim results from a global comparative-effectiveness observational study. Annual Scientific Meeting of the American College of Rheumatology, 2011, Chicago. Arthritis Rheum 2011:63:S45.

8. Buch MH. Sequential use of biologic therapy in rheumatoid arthritis. Curr Opin Rheumatol 2010;22:321-9.

9. Kekow J, Mueller-Ladner U, Link R, et al. Real life treatment with rituximab in TNF blocker non-responders is superior to treatment with a second TNF blocker. Annual Scientific Meeting of the American College of Rheumatology (ACR), 2010, Atlanta. Arthritis Rheum 2010;\$406.

10. Ancuta I, Codreanu C, lonescu R, et al. Rituximab after TNF inhibitors — analysis of real life RA treatment practice. Annual European Congress of Rheumatology, 2011, London. Ann Rheum Dis 2011;69:FRI0360.

11. Alivernini S, Laria A, Gremese E, et al. ACR70-disease activity score remission achievement from switches between all the available biological agents in rheumatoid arthritis: a systematic review of the literature. Arthritis Res Ther 2009;11:R163.

12. van der Heijde DM. Radiographic imaging: the 'gold standard' for assessment of disease progression in rheumatoid arthritis. Rheumatology (Oxford) 2000;39(Suppl 1):9-16.

13. Landewé $\mathbf{R}$, van der Heijde D, Klareskog L, et al. Disconnect between inflammation and joint destruction after treatment with etanercept plus methotrexate: results from the trial of etanercept and methotrexate with radiographic and patient outcomes. Arthritis Rheum 2006;54:3119-25.

14. Cohen SB, Emery P, Greenwald MW, et al. Rituximab for rheumatoid arthritis refractory to anti-tumor necrosis factor therapy: results of a multicenter, randomized, double-blind, placebo-controlled, phase III trial evaluating primary efficacy and safety at twenty-four weeks. Arthritis Rheum 2006;54:2793-806.

15. van der Heijde DM. Overview of radiologic efficacy of new treatments. Rheum Dis Clin North Am 2004;30:285-93, vi.

16. Keystone $\mathbf{E}$, Emery P, Peterfy CG, et al. Rituximab inhibits structural joint damage in patients with rheumatoid arthritis with an inadequate response to tumour necrosis factor inhibitor therapies. Ann Rheum Dis 2009;68:216-21.

17. Uitz $\mathbf{E}$, Fransen J, Langenegger T, et al. Clinical quality management in rheumatoid arthritis: putting theory into practice. Swiss Clinical Quality Management in Rheumatoid Arthritis. Rheumatology (Oxford) 2000:39:542-9.

18. Kobelt G, Kasteng R. Access to innovative treatments in rheumatoid arthritis in Europe: uptake of biologic treatments. Report for the European Federation of Pharmaceutical Industry Associations (EFPIA), 2009. http://www.efpia.eu/Content/ Default.asp?PagelD $=5598 D o c \mid D=7640$ (accessed date 8 August 2011).

19. Dixon WG, Carmona L, Finckh A, et al. EULAR points to consider when establishing, analysing and reporting safety data of biologics registers in rheumatology. Ann Rheum Dis 2010;69:1596-602.

20. Rau R, Wassenberg S, Herborn G, et al. A new method of scoring radiographic change in rheumatoid arthritis. J Rheumatol 1998;25:2094-107.

21. Creemers $\mathbf{M}$, Fransen J, Van Riel P. Reliability and sensitivity to change of the Ratingen joint damage score in a cohort of early RA patients. Annual European Meeting of Rheumatology, 2006, Amsterdam. Ann Rheum Dis 2006;S165.

22. Loiero D. Inter- and Intrareader Variability of Ratingen Score in the SCOM Database and Its Causes. Zurich: University of Zurich, 2011.

23. Fries JF, Spitz P, Kraines RG, et al. Measurement of patient outcome in arthritis. Arthritis Rheum 1980;23:137-45.

24. Finckh A, Simard JF, Duryea J, et al. The effectiveness of anti-tumor necrosis factor therapy in preventing progressive radiographic joint damage in rheumatoid arthritis: a population-based study. Arthritis Rheum 2006;54:54-9.

25. Wells GA, Tugwell P, Kraag GR, et al. Minimum important difference between patients with rheumatoid arthritis: the patient's perspective. J Rheumatol 1993;20:557-60.

26. O'Dell JR. Therapeutic strategies for rheumatoid arthritis. N Engl J Med 2004;350:2591-602.

27. Chatzidionysiou K, Lie E, Nasonov E, et al. Highest clinical effectiveness of rituximab in autoantibody-positive patients with rheumatoid arthritis and in those for whom no more than one previous TNF antagonist has failed: pooled data from 10 European registries. Ann Rheum Dis 2011;70:1575-80.

28. de Punder YM, van Riel PL. Rheumatoid arthritis: understanding joint damage and physical disability in RA. Nat Rev Rheumatol 2011;7:260-1. 